\title{
Toward a second order description of neuronal networks Duane Q Nykamp
}

\author{
Address: School of Mathematics, University of Minnesota, MN, USA
}

Email: Duane Q Nykamp - nykamp@math.umn.edu

from Eighteenth Annual Computational Neuroscience Meeting: CNS*2009

Berlin, Germany. 18-23 July 2009

Published: I 3 July 2009

BMC Neuroscience 2009, I0(Suppl I):PI68 doi:I0.I|86/I47|-2202-I0-SI-PI68

This abstract is available from: http://www.biomedcentral.com/I47I-2202/I0/SI/PI68

(c) 2009 Nykamp; licensee BioMed Central Ltd.

The complexity of the activity of large numbers of neurons and their interconnectivity creates a challenge for understanding computations within the brain. The high dimensionality of the activity patterns and connectivity patterns, combined with the difficulty of estimating them from limited data sets, is a tremendous obstacle to an analysis of the relationships between the connectivity and the behavior of the network.

To address this complexity, I present a kinetic theory approach toward distilling complicated network connectivity into a simplified description of network features and then estimating what network behaviors result from those features. Moreover, to connect the theoretical analysis with experiment, I present a connectivity analysis through which one may be able to constrain estimates of the simplified network features from experimental data.

I demonstrate evidence that one can greatly reduce the dimension required to represent network connectivity patterns by using a kinetic theory approach that neglects higher order statistics of those patterns. By parameterizing connectivity patterns with their first and second order statistics, I illustrate a method to distill the connectivity down to its key features and study how these features influence network behavior.

This kinetic theory modeling approach is based on certain first and second order statistics of connectivity patterns. I have developed a connectivity analysis to estimate such connectivity statistics from experimental data while explicitly controlling for effects of hidden neurons. By linking the simplified network analysis to network struc- ture in the brain, the connectivity analysis combined with the kinetic theory analysis could prove to be a powerful combination for probing relationships between network connectivity patterns and behavior.

\section{Acknowledgements}

This work was supported by in part by NSF grant DMS 07/9724. 